\title{
Resultados econômicos e funcionais na cirurgia para hérnia do disco lombar
}

\author{
Jefferson Walter Daniel", Fabiane de Araújo Cesare ${ }^{\star \star}$, Luciano Haddad ${ }^{\star \star \star}$, Antônio Barros \\ Carreira $^{\star \star \star}$, Luiz Fernando Cannoni* ${ }^{\star \star \star}$, José Carlos Esteves Veiga ${ }^{\star \star \star \star}$ \\ Disciplina de Neurocirurgia do Departamento de Cirurgia da Faculdade de Ciências Médicas da Santa Casa de São Paulo, \\ São Paulo
}

\section{RESUMO}

O objetivo deste estudo é a comparação dos resultados cirúrgicos entre pacientes beneficiados por compensações financeiras previdenciárias decorrentes da inatividade ocupacional e aqueles que não as recebem, antes e após terem sido operados de hérnia do disco lombar.

Esta análise retrospectiva abrangeu o periodo de fevereiro de 1999 a janeiro de 2002. Foram estudados 47 pacientes consecutivos operados de hérnias discais lombares de etiologia degenerativa e sem artrodese, no Serviço de Neurocirurgia da Santa Casa de São Paulo. Foram analisados prontuários hospitalares e mantidos contatos telefônicos com os pacientes. Aplicamos questionário padronizado com informações a respeito de: estado clínico, ocupações, esforços fisicos e recebimento de beneficios financeiros antes e após a cirurgia. Utilizamos a escala econômico-funcional de Prolo para a avaliação dos resultados obtidos. Todos os doentes apresentavam sinais e sintomas semelhantes no periodo pré-operatório.

Os pacientes que recebem regularmente compensações financeiras previdenciárias, quando comparados àqueles sem benefícios, permaneceram inativos por periodo, em média, $69 \%$ maior; obtiveram apenas $21 \%$ de resultados excelentes e bons, apesar dos mesmos esforços físicos ocupacionais e $100 \%$ não retornaram ao trabalho. Houve diferença estatisticamente significativa entre o tempo de inatividade pré e pós-operatória dos pacientes que receberam benefício e o daqueles que não receberam beneficio ( $p<0,001$, Mann-Whitney), e também nos valores da escala de Prolo entre os dois grupos ( $p<0,001$, teste $t)$. Vários fatores influenciaram no resultado clínico final, em especial a seguridade social na forma de sua assistência beneficiária.

\section{PALAVRAS-CHAVE}

Hérnia do disco lombar. Resultados cirúrgicos. Benefícios financeiros previdenciários.

\section{ABSTRACT}

Economical and functional results for herniated lumbar disc surgery

This study's aim is to compare surgical results among patients receiving and not receiving working inactivity financial grants, before and after they were operated for herniated lumbar discs.

A retrospective analysis of 47 consecutive patients operated on for degenerative herniated discs without arthrodesis, between February, 1999 and January, 2002, at the Neurosurgical Service of Hospital da Santa Casa, São Paulo, Brazil. Patient's hospital medical records were analyzed and telephone interviews were made by applying a previous standard questionnaire to them, containing information such as clinical status, occupations, physical strains and the fact of receiving or not financial grants before and after surgery, using Prolo's economic-functional scale to evaluate results.

Similar signs and symptoms were present in all patients in the pre-operative period, but when those receiving financial grants were compared to those not receiving, the first group of patients stayed away from work $69 \%$ longer, had only $21 \%$ of excellent and good results were observed, even though their occupational strains were similar and $100 \%$ did not go back to their prior occupation. There was a statistical difference among patients receiving or not the working inactive financial grants before and after surgery ( $p<0.001$, Mann-Whitney) and Prolo's scales values between these two groups $(p<$ 0.001 , test $t$ ). Many factors participate in the final clinical results just as social security assistance benefits.

\section{KEYWORDS}

Lumbar herniated disc. Surgical results. Social financial grants.

\footnotetext{
*Professor Instrutor da Disciplina de Neurocirurgia da Faculdade de Ciĉncias Médicas da Santa Casa de São Paulo.

**Residente de terceiro ano do Serviço de Neurocirurgia da Irmandade da Santa Casa de Misericórdia de São Paulo.

***Médico Assistente do Serviço de Neurocirurgia da Irmandade da Santa Casa de Misericórdia de São Paulo.

**** Professor Adjunto Doutor da Disciplina de Neurocirurgia da Faculdade de Ciências Médicas da Santa Casa de São Paulo c Chefe do Serviço.
} 


\section{Introdução}

Os resultados considerados bons e excelentes ocorrem em $75 \%$ a $96 \%$ das cirurgias para hérnia do disco lombar. Porém, quando há compensação financeira pela inatividade do trabalho, verifica-se grau de insatisfação com o tratamento cirúrgico em $42 \%$ a $72 \%$ dos casos ${ }^{1,4,13}$. O reconhecimento desse fato foi relatado por Spurling e Grantham. ${ }^{16}$, em 1949, e confirmado por outros autores posteriormente $e^{4,8,10,13}$.

Indicadores prognósticos sugerem resultados regulares ou ruins quando os candidatos à cirurgia apresentam idade acima dos 50 anos, dor lombar crônica e persistente ${ }^{5}$, dor ciática contínua por período maior que oito meses ${ }^{12}$, insatisfação e afastamento do trabalho, ganho de beneficios previdenciários, somatização psicológica ${ }^{\mathrm{It}}$ e doenças musculoesqueléticas associadas, tais como a síndrome miofascial e a espondiloartrose ${ }^{3}$.

Apesar de explanações exaustivas ao paciente, aos seus familiares e indicação cirúrgica criteriosa, o paciente pode apresentar incapacidade para conviver com possíveis seqüelas inerentes à doença degenerativa da coluna lombar. Motivos para processos de indenizações jurídicas em suas várias formas são: a dor e o sofrimento residual, déficits neurológicos, dúvidas e questionamentos não esclarecidos, além de diagnóstico e tratamento inadequados ${ }^{7}$.

O objetivo deste estudo é analisar os resultados cirúrgicos da hérnia do disco lombar comparando doentes que recebem compensações financeiras previdenciárias por inatividade ocupacional com aqueles que não as recebem.

\section{Casuística e métodos}

O período de estudo estendeu-se de fevereiro de 1999 a janeiro de 2002. Foram analisados, retrospectivamente, 50 pacientes consecutivos tratados no Serviço de Neurocirurgia da Santa Casa de São Paulo pela cirurgia convencional para hérnia do disco lombar de etiologia degenerativa como causa principal de sua sintomatologia. Foram excluídos três pacientes em virtude da insuficiência de dados e analisados 47 casos mediante revisão de prontuários hospitalares e de entrevistas telefônicas encerradas em novembro de 2002.

Um questionário padronizado aplicado aos pacientes avaliou o estado clínico atual, atividades habituais e profissionais prévias e após a cirurgia, esforços físicos realizados em suas ocupações (quantificados pelos entrevistados) e o fato de receberem ou não compensaçōes financeiras decorrentes da inatividade ocupacional (Quadro 1).

\section{Quadro 1}

Capacidade econômica e funcional prévia e após a cirurgia (questionário aplicado por contato telefônico)

1. Estado clínico, atividades econômicas e hábitos sociais utilizando-se a escala econômico-funcional de Prololit

2. Atividades profissionais: esforços físicos realizados quantificados pelo paciente em pesado, moderado e leve; relação trabalhista qualificada pelo paciente em assalariado, autônomo, do lar, desempregado ou afastado 3. Ganho ou não de benefícios financeiros previdenciários

Foram comparados os pacientes que recebem benefícios com aqueles que não os recebem, utilizandose a escala econômico-funcional proposta por $\mathrm{Prolo}^{14}$ (Quadro 2).

Quadro 2

Escala econômico-funcional de Prolo ${ }^{14}$

\begin{tabular}{l} 
Escala econômica \\
$\bar{E} 1$ : invalidez completa \\
E2: inabilidade de realizar atividades produtivas, incluindo trabalhos \\
domésticos \\
E3: inabilidade de trabalhar em ocupação prévia \\
E4: limitação no desenvolvimento de trabalho em sua ocupação profissional \\
E5: trabalha em ocupação prévia sem restrições \\
Escala funcional \\
\hline F1: incapacidade total ou pior que antes da cirurgia \\
F2: dor lombar e/ou ciática leve a moderada ou igual a antes da ci- \\
Furgia, porém capaz de realizar atividades diárias \\
F4: dor leve a moderada capaz de realizar todas as atividades, exceto \\
F5: dor lombuperação completa, ausência de episódios recorrentes de dor \\
lombar e/ou ciática, capaz de realizar atividades desportivas prévias à \\
cirurgia
\end{tabular}

Resultados segundo o somalório dos pontos: Ruim $=2-4$; Regular $=5-6 ;$ Bom $=7-8$; Excelentc $=9-10$.

$\mathrm{Na}$ avaliação pré-operatória, foram solicitadas radiografias simples dinâmicas da coluna lombar - para o estudo da estabilidade segmentar - e ressonância magnética, a princípio, sem contraste. Foi utilizado contraste paramagnético nos casos com hérnia discal recorrente ou de processo inflamatório reacional local. $\mathrm{Na}$ hipótese de espondilólise ou espondilolistese, o estudo foi complementado pela tomografia axial computadorizada da coluna lombar com janela óssea.

Todos os pacientes foram submetidos à discectomia convencional sem artrodese, com o auxílio do microscópio cirúrgico, sob anestesia geral e em decúbito ventral. O procedimento teve duração aproximada de duas horas para hérnias únicas. $\mathrm{O}$ antibiótico utilizado 
foi a ceftriaxona, iniciada duas horas antes do procedimento e mantida por 48 horas, conforme orientação da Comissão de Infecção Hospitalar.

\section{Resultados}

A média de idade dos 47 pacientes operados foi de 45 anos (mínimo de 20 e máximo de 78 anos), sendo $33(70,21 \%)$ masculinos e $14(29,21 \%)$ femininos.

O período médio de acompanhamento ambulatorial foi de 13,70 meses (mínimo de 0 e máximo de 39 meses).

A duração da sintomatologia pré-operatória foi, em média, de 37,23 meses (com os limites de variação entre um mês e cinco anos). A queixa inicial foi de lombociatalgia em $61,70 \%$ dos casos, lombalgia em $25,53 \%$, ciatalgia em $6,38 \%$ e outros sintomas em $6,38 \%$. A queixa principal também foi lombociatalgia, ocorrendo em $85,10 \%$ dos casos, seguidos por ciatalgia em $6,38 \%$, outros sintomas em $6,38 \%$ e lombalgia em 2,1\% (Gráfico 1).

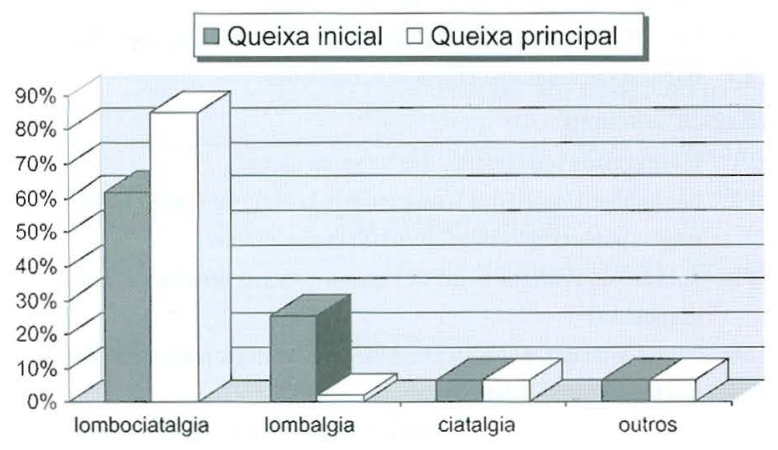

Gráfico 1 - Distribuição de freqüência das queixas inicial e principal no periodo pré-operatório em 47 pacientes.

Não houve diferença estatística entre as queixas iniciais dos pacientes que recebiam benefício e as dos pacientes que não recebiam auxílio previdenciário ( $p>0,05$, teste do qui-quadrado).

As síndromes de compressão dos nervos espinhais (déficit motor, sensitivo, alterações dos reflexos) em várias combinações ocorreram em 100\% dos pacientes e o sinal de Lasègue era presente em $65,95 \%$ dos casos.

O período médio de internação pós-operatória foi de 4,12 dias (mínimo de 1 e máximo de 26 dias).

Os níveis segmentares operados foram: L1-L2 em 1 (1,96\%); L3-L4 em 4 (7,84\%); L4-L5 em 18 (35,29\%); L5-S1 em 28 (54,90\%). Discectomia em múltiplos níveis ocorreu em três pacientes $(6,38 \%)$ para hérnias simultâneas em L4-L5 e L5-S1 em dois pacientes $(66,66 \%)$ e L3-L4, L4-L5 e L5-S1 em um paciente (33,33\%). Três pacientes foram reoperados. Em um dos casos, a reoperação ocorreu 20 dias após a primeira cirurgia em decorrência de disco herniado recorrente no mesmo nível segmentar. Este paciente continua afastado de sua ocupação, a qual era considerada pesada. Recebe benefícios financeiros previdenciários há dois anos e 0 seu resultado clínico é ruim (Prolo 4). O paciente seguinte foi operado três vezes, a primeira cirurgia havia sido há cinco anos (antes desta série), a segunda há três anos em virtude de hérnia recorrente no mesmo nível segmentar e a terceira cirurgia há dois anos, em decorrência de cisto meníngeo por trauma cirúrgico no mesmo nível. Persiste afastado do trabalho, o qual era considerado pesado, $\mathrm{e}$ com resultado ruim (Prolo 4). O terceiro paciente havia sido operado há três anos e reoperado um mês após a primeira intervenção devida à hérnia recorrente; não obteve benefícios previdenciários, retornou à sua ocupação profissional, considerada leve, e com resultado excelente (Prolo 10).

Beneficios financeiros previdunciários decorrentes da inatividade do trabalho eram recebidos por $19(40,42 \%)$ pacientes, dos quais 100\% não tinham ocupaçôes econômicas por ocasião deste estudo. Destes 19 pacientes, todos tinham vínculos empregatícios e eram assalariados antes da cirurgia. Nos demais $28(59,57 \%), 21(75 \%)$ trabalhavam e $7(25 \%)$ não, 5 por estarem desempregados e 2 "afastados". Dos 21 pacientes que retornaram às suas ocupações após a cirurgia, 15 são autônomos, 4 do lar e 2 assalariados. Dos 19 pacientes com compensações financeiras, $9(47,36 \%)$ julgaram o esforço físico realizado em sua ocupação profissional pesado, $8(42,10 \%)$ moderado e $2(10,52 \%)$ leve. Nos 28 pacientes sem compensações financeiras, $10(35,71 \%)$ julgaram as suas ocupações pesadas, $11(39,28 \%)$ moderada e $7(25 \%)$ leve (Tabela 1).

\section{Tabela 1}

Distribuição de freqüência entre os grupos com e sem beneficio financeiro previdenciário em relação aos esforços físicos no trabalho

\begin{tabular}{lcc}
\hline Esforço físico & $\begin{array}{c}\text { Com benefício } \\
\mathbf{N}=\mathbf{1 9}(\mathbf{4 0 , 4 2 \% )}\end{array}$ & $\begin{array}{c}\text { Sem benefício } \\
\mathbf{N = 2 8}(\mathbf{5 9 , 5 7 \% )}\end{array}$ \\
\hline Leve & $2(10,52 \%)$ & $7(25 \%)$ \\
Moderado & $8(42,10 \%)$ & $11(39,28 \%)$ \\
Pesado & $9(47,36 \%)$ & $10(35,71 \%)$ \\
\hline
\end{tabular}

No tocante ao período de inatividade ocupacional, nos pacientes com benefícios antes da cirurgia foi, em média, de 12,52 meses no pré-operatório e 22,94 meses no pós-operatório. Já naqueles sem benefícios, foi de 5,39 e 5,64 meses respectivamente (Tabela 2). Houve diferença estatística entre o período de inatividade pré- 


\section{Tabela 2}

Período médio de inatividade ocupacional pré e póscirúrgico dos pacientes com e sem beneficios financeiros previdenciários

\begin{tabular}{lcc}
\multicolumn{2}{c}{ Inatividade } & \multicolumn{2}{c}{ Tempo de inatividade ocupacional } \\
Benefício & Pré-cirúrgico & Pós-cirrurgico \\
\hline Com benefícios & 12,52 meses & 22,94 meses \\
Sem benefícios & 5,39 meses & 5,64 meses \\
\hline \hline
\end{tabular}

operatório dos pacientes que recebiam benefício previdenciário e o daqueles sem benefícios $(\mathrm{p}=0,047$, teste Mann-Whitney). Houve ainda diferença no tempo de inatividade no pós-operatório entre o grupo com benefício, média de 22,94 meses, e o grupo sem benefício previdenciário, média de 5,64 meses ( $p<$ 0,001 , teste $t$ ) (Gráfico 2).

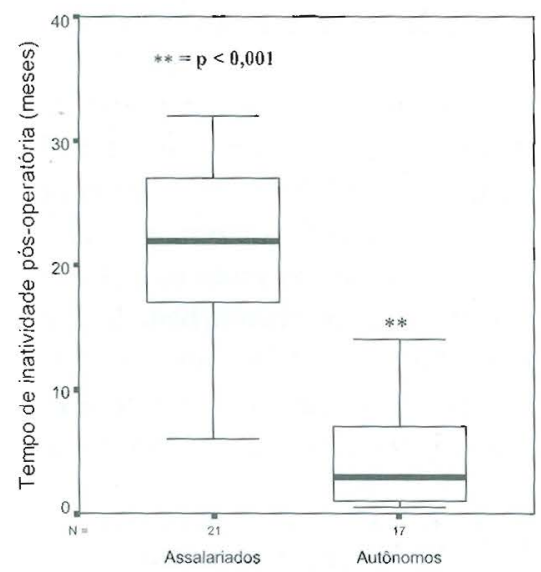

Gráfico 2 - Tempo de inatividade pós-operatória (em meses) entre os pacientes assalariados (com benefícios previdenciários) e os pacientes autônomos (sem beneficios previdenciários).

A escala econômico-funcional de Prolo ${ }^{14}$ correlaciona o bem-estar funcional e social com o desempenho econômico e produtivo no período pré e pós-operatório. Os resultados foram analisados no grupo geral e nos grupos com e sem benefícios financeiros representados no gráfico 3. Houve diferença estatística entre o valor da escala de Prolo dos pacientes que recebiam benefício, média de 5,09, e a dos pacientes que não recebiam benefício, média de $7,88(\mathrm{p}<0,001$, teste $t)$ (Gráfico 4).

\section{Discussão}

\section{Aspectos clínicos}

O período médio de 13,7 meses de acompanhamento pós-operatório neste estudo permite avaliar

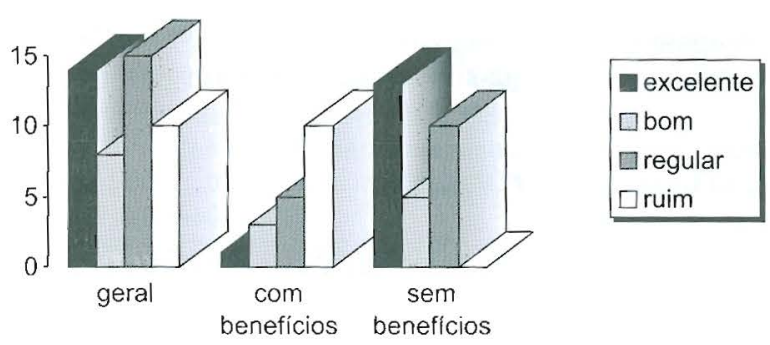

Gráfico 3 - Distribuição de frequiência dos resultados cirúrgicos pela escala de Prolo 14 em 47 pacientes operados.

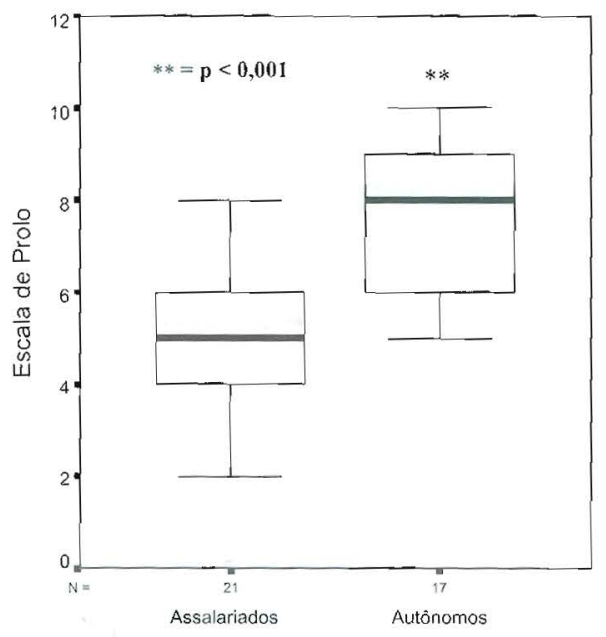

Gráfico 4-Escala de Prolo entre os pacientes assalariados (com benefícios previdenciários) e os pacientes autônomos (sem beneficios previdenciários).

a tendência da evolução clínica como sugerido por

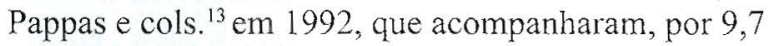
meses, 654 casos de hérnia discal lombar tratados cirurgicamente. Porém, períodos mais prolongados de seguimento, como o publicado por Davis ${ }^{4}$, em 1994, de 10,8 anos, permitem conclusões mais abrangentes. O predomínio do sexo masculino e da faixa etária média de 45 anos de idade são achados similares em todos as séries estudadas ${ }^{4,5,10,13,16,17}$.

Lombociatalgia foi a queixa inicial e principal em $61,70 \%$ e $85,10 \%$ dos casos respectivamente. Síndromes dos nervos espinhais, únicas ou associadas, ocorreram em $100 \%$ dos pacientes e o sinal de Lasègue presente em até 60 graus de elevação do membro inferior ocorreu em $65,95 \%$ dos casos.

Durante a realização do inquérito preliminar, pudemos constatar que não houve diferença quanto à sintomatologia pré-operatória entre pacientes com piores resultados e recebendo compensações financeiras e aqueles com melhores resultados e trabalhando ( $p>0,05$, teste do qui-quadrado). Dvorak e cols. ${ }^{5}$ sugerem fatores sociais, psicológicos e ganhos secundários para explicar os motivos destes piores 
resultados no grupo com benefícios financeiros, mesmo com sintomatologia similar antes da cirurgia.

\section{Aspectos cirúrgicos}

A sintomatologia é decorrente de uma única hérnia de disco na maioria dos casos, havendo a tendência para a exploração de outros níveis no ato operatório quando o achado do primeiro não é convincente ${ }^{15}$. Em nossa série, um nível segmentar foi operado em $93,61 \%$ dos pacientes e, nos demais, dois e três níveis, com resultado ruim (Prolo 4) e regular (Prolo 6) em dois pacientes e bom (Prolo 8) em um.

Quanto maior for o período de seguimento clínico, maiores são as possibilidades de reoperações, conforme demonstrado por Davis ${ }^{4}$ ao relatar que $33 \%$ dos seus 984 pacientes foram reoperados após quatro anos. Reoperações devidas a discos recorrentes, fístulas liquóricas, infecções e outras causas ocorrem em $3 \%$ a $18 \%$ dos $\operatorname{casos}^{1,10,13,15}$. O baixo índice de reintervenções $(6,38 \%)$ ocorridas em nossa série se deve, possivelmente, ao curto período de seguimento pósoperatório.

\section{Ocupações}

Os pacientes que apresentam dor crônica e recebem benefícios previdenciários têm menores possibilidades de retornar ao trabalho em relação aos que não recebem benefícios, apesar do estado clínico similar ${ }^{6,8}$. O mercado de trabalho naturalmente irá selecionar os melhores funcionários para as ocupações pretendidas relacionadas a aspectos de capacitação profissional, de saúde, grau de escolaridade e posição social, sendo óbvia a constatação, na prática médica diária, de que o receio de perder o beneficio decorrente da inatividade ocupacional o faz permanecer incapacitado para não retornar ao seu trabalho e com a possibilidade de perder seu vínculo empregatício. Não existem estatísticas oficiais em nosso país determinando quantos pacientes estão afastados e recebendo benefícios previdenciários no período pós-operatório de hérnia do disco lombar. Conforme o sistema de seguridade social, assistência à saúde e características regionais de cada país, pode haver maior ou menor dificuldade no direito de receber benefícios da previdência social, influenciando, assim, os resultados do tratamento cirúrgico da hérnia do disco lombar².

Ocupações que exigem grandes esforços físicos apresentam funcionários com maiores incidências de disfunções musculoesqueléticas, em especial lombalgia seguida por doenças do joelho ${ }^{3}$. Outros fatores participam no processo de incapacidade funcional além dos esforços físicos, tais como ambiente de trabalho, outras doenças associadas, hábitos de vida e problemas psicológicos ${ }^{9}$. Nos indivíduos cujas atividades profissionais exigem esforços físicos, ocorrem piores resultados pós-operatórios, mesmo sem ganhos secundários $^{4}$. Em nosso estudo, dos 19 pacientes recebendo benefícios financeiros antes e/ou após a cirurgia, $89,46 \%$ tinham ocupações nas quais esforços físicos pesados e moderados eram realizados, obtendose resultados cirúrgicos ruins e regulares em 78,99\% destes casos. Entre os 28 pacientes sem benefícios, $74,99 \%$ faziam os mesmos esforços pesados e moderados, porém apresentaram resultados cirúrgicos ruins e regulares em apenas $35,71 \%$ dos casos. Houve diferença estatística significativa dos resultados pósoperatórios de pacientes que desempenhavam trabalho pesado ou moderado entre o grupo que recebia benefícios financeiros e o grupo que não recebia benefícios ( $\mathrm{p}<0,001$, teste de qui-quadrado).

O período de inatividade ocupacional antes e após a cirurgia tende a ser maior em pacientes recebendo benefícios financeiros, mesmo com melhora clínica da dor, provavelmente por falta de motivos em deixar de receber a compensação financeira previdenciária ${ }^{17}$. Possivelmente, são esses os motivos pelos quais, em nossa série, os pacientes recebendo benefícios estiveram inativos, em média, por 12,52 meses antes da cirurgia e 22,94 meses após a cirurgia, em comparação àqueles sem benefícios, 5,39 e 5,64 meses, respectivamente.

\section{Escala de Prolo}

A escala de graduação econômico-funcional proposta por Prolo' ${ }^{14}$, em 1986, justifica-se pelas seguintes características: simplicidade e praticidade em seu uso; incluir elementos essenciais de resposta do paciente ao tratamento; ser menos subjetiva do que outros métodos; ser semiquantitativa; e poder ser correlacionada com dados anatômicos. A intenção desta escala é a de avaliar e expressar resultados clínicos de procedimentos cirúrgicos para hérnias discais lombares e comparar o estado econômico e funcional de populações de pacientes antes e após as cirurgias. Em nossa série, resultados excelentes e bons ocorreram em $46,80 \%$ dos 47 pacientes, porém, quando avaliados separadamente em grupos de pacientes recebendo e não recebendo benefícios, esses índices demonstram diferenças significativas, 21,04\% e $64,27 \%$, respectivamente. A diferença dos valores médios destes dois grupos é maior do que seria esperado por acaso, havendo diferença estatística significativa $(\mathrm{p}<0,001$, test $t)$. Não há paralelo exato deste estudo com outros na literatura, porém o índice de sucesso ocorre em $75 \%$ a $96 \%$ nas mencionadas séries. Já em pacientes com pendências legais e benefícios previdenciários, a taxa de bons e excelentes resultados variou de $42 \%$ a $72 \%$. 


\section{Conclusões}

Não houve diferença estatística entre as queixas iniciais e principais dos doentes que recebiam benefícios previdenciários e as daqueles que não os recebiam ( $p>0,05$, teste do qui-quadrado).

$\mathrm{O}$ período de inatividade ocupacional prévia e posterior à cinurgia foi maior nos pacientes com auxílios financeiros previdenciários em relação àqueles sem o auxílio ( $\mathrm{p}=0,047$, teste Mann-Whitney e $\mathrm{p}<0,001$, teste $t$, respectivamente).

Piores resultados cirúrgicos ocorreram nos doentes recebendo benefícios financeiros previdenciários $\mathrm{e}$ exercendo trabalhos pesados ou moderados em relação aos doentes com os mesmos esforços e sem benefícios ( $\mathrm{p}<0,001$, teste do qui-quadrado).

Resultados excelentes e bons são menos freqüentes em doentes que recebem benefícios financeiros previdenciários.

Todos os doentes que recebiam benefícios financeiros previdenciários permaneceram afastados do trabalho após a cirurgia.

\section{Referências}

1. ASH HL, LEWIS PJ, MORELAND DB, EGNATCHIK JG, YU YJ, CLABEAUX DE, HYLAND AH: Prospective multiple outcomes study of outpatient lumbar microdiscectomy: should 75 to $80 \%$ success rates be the norm? J Neurosurg (Spine) 96:34-44, 2002.

2. CHERKIN DC, DEYO RA, LOESER JD, BUSH T, WADDELL G: An international comparison of back surgery rates. Spine 19:1201-6, 1994.

3. CUNNINGHAM LS, KELSEY JL: Epidemiology of muscloskeletal impairments and associated disability. Am J Publ Health 74:574-9, 1984.

4. DAVIS RA: A long-term outcome analysis of 984 surgically treated herniated lumbar discs. J Neurosurg 80:415-21, 1994.

5. DVORAK JK, GAUCHAT MH, VALACH L: The outcome of surgery for lumbar sisc hemiation. Spine 13:1418-22, 1988.
6. DWORKIN RH, HANDLIN DS, RICHLIN DM, BRAND L, VANNUCCI C: Unraviling the effects of compensation, litigation and employment on treatment response in chronic pain. Pain 23:49-59, 1985.

7. EPSTEIN N: It is easier to confuse a jury than convince a judge: the crises in medical malpractice. Spine 27:2425-30, 2002.

8. GREENOUGH CG, FRASER RD: The effects of compensation on recovery from low-back injury. Spine 14:947$55,1989$.

9. KARE H, KRISTIAN T, TOR B: A prospective cohort study of risk factors for disability retirement because of back pain in the general working population. Spine 27:17906, 2002.

10. LEWIS PJ, WEIR BKA, BROAD RW, GRACE MG: Longterm prospective study of lumbosacral discectomy. J Neurosurg 67:49-53, 1987.

11. NICKEL R, EGLE UT, EYSEL P, ROMPE JD, ZOLLNER J. HOFFMANN SO: Health-related quality of life and somatization in patientes with long-term low back pain. Spine 26:2271-7, 2001.

12. NYGAARD OP, KLOSTER R, SOLBERG T: Duration of leg pain as a predictor of outcome after surgery for lumbar disc herniation: a prospective cohort study with 1-year follow up. J Neurosurg (Spine) 92:131-4, 2000.

13. PAPPAS CTE, HARRINGTON T, SONNTAG VKH: Outcome analysis in 654 surgically treated lumbar disc heriations. Neurosurgery 30:862-6, 1992.

14. PROLO DJ, OKLUND SA, BUTCHER M: Toward uniformity in evaluating results of lumbar spine operations. Spine 11:601-6, 1986.

15. SILVERS HR: Microsurgical versus standard lumbar discectomy. Neurosurgery 22:837-41, 1988.

16. SPURLING RG, GRANTHAM EG: The end-results of surgery for ruptured lumbar intervertebral discs. J Neurosurg 6:57-64, 1949.

17. WEBER H: Lumbar disc herniation. Spine 8:131-40, 1983.

Original recebido em julho de 2004

Aceito para publicação em outubro de 2004

\author{
Endereço para correspondência: \\ Jefferson Walter Daniel \\ Rua Tiapira, 264 \\ CEP 05578-000 - São Paulo, SP \\ E-mail: dolldani@uol.com.br
}

